\title{
Comparison of clinic-based versus home-based balance and agility training for the symptoms of knee osteo- arthritis
}

\author{
Matthew W Rogers (MS) ${ }^{1}$ \\ Nauris Tamulevicius (PhD) ${ }^{2}$ \\ Stuart J Semple (PhD) ${ }^{1}$ \\ Marius F Coetsee (PhD) ${ }^{1}$ \\ Beth F Curry (BS) ${ }^{3}$ \\ ${ }^{1}$ Department of Biokinetics \& Sport Science, University of Zululand, KwaDlangezwa, KwaZulu-Natal, South Africa \\ ${ }^{2}$ School of Human Performance and Leisure Sciences, Barry University, Miami Shores, Florida, USA \\ ${ }^{3}$ Cheek-Powell Wellness Center, Morton Plant Mease Healthcare, Clearwater, Florida, USA
}

Correspondence to: Stuart Semple (ssemple@pan.uzulu.ac.za)

\begin{abstract}
Objective. To compare clinic-based (CB) and home-based (HB) deliveries of a knee osteoarthritis (OA) exercise programme.

Methods. Outcomes from a $\mathrm{CB}$ exercise study $(N=6)$ utilising kinesthesia, balance and agility (KBA) exercises were compared with those from a HB KBA study $(N=6)$. Both conditions trained 30 minutes, 3 days per week for 8 weeks. CB sessions were conducted in a group led by an exercise physiologist (EP); HB participants received an initial 3 sessions of one-to-one training from an EP, written/pictorial instructions, telephone and e-mail follow-up, and in-person refresher sessions during weeks 4 and 6 . The primary outcome was an OA-specific physical function survey. Community activity level, self-report knee stability, 15-m get up and go walk, and stair climb and descent were also measured.

Results. Adherence was $94 \%$ in both conditions. KBA improved $\mathrm{PF}$ in both CB $(59 \% ; 18 \pm 12.5 \mathrm{pts} ; p=0.008)$ and $\mathrm{HB}(33 \% ; 7.3 \pm 7.5$ pts; $p=0.03$ ), with no difference between conditions. All outcome improvements were somewhat larger for $\mathrm{CB}$, but these differences did not reach statistical significance.

Conclusion. We found no difference in outcomes between $\mathrm{CB}$ and $\mathrm{HB}$ exercise in this preliminary comparison. Our results support that KBA is an effective intervention for symptomatic knee $O A$ that may be delivered in $\mathrm{CB}$ or $\mathrm{HB}$ settings.
\end{abstract}

\section{Introduction}

Osteoarthritis $(\mathrm{OA})$ of the knee is a common cause of pain, stiffness and physical impairment in adults. The lifetime risk of developing symptomatic knee OA is nearly $45 \%$. In the USA radiographic knee $\mathrm{OA}$ is estimated to be present in $37 \%$ of people over 60 years of age, with symptomatic knee OA affecting $12 \%$ of that age group. ${ }^{2}$ While the number of knee arthroplasties is expected to rise dramatically in the coming years, ${ }^{3-5}$ many patients continue to seek non-surgical relief. While there are no disease-modifying treatments, there is good evidence for the efficacy of various exercise interventions to improve pain and function among persons with knee OA. ${ }^{6}$
Kinesthesia, balance and agility exercise (KBA) is a neuromuscular training programme designed to improve dynamic joint stability and neuromuscular control. KBA challenges the vestibular, visual and somatosensory systems (with adaptations generally occurring only in the somatosensory system). ${ }^{7}$ Such programmes employ agility walking drills, e.g. tandem walking, grapevine, side-stepping and balance challenge activities. Given the often poor dynamic joint stability and neuromuscular control associated with knee $\mathrm{OA},{ }^{8-10}$ programmes that incorporate KBA have been employed as an intervention. ${ }^{11-15}$ While not yet researched extensively, KBA appears to be a promising functional treatment for persons with knee OA. Fitzgerald and colleagues ${ }^{11}$ reported a case study of a 73-yearold female patient with dynamic knee instability from bilateral knee OA. KBA training and traditional therapeutic exercise were combined twice per week for 6 weeks, resulting in the patient's return to golf and tennis and an ability to walk and climb stairs without knee instability. In another study, the authors of an 8-week, 3 times per week clinical trial $^{13}$ concluded that the addition of KBA exercises had added benefits over strength training alone on all functional outcomes measured. Two studies investigated the effects of KBA independent of other therapeutic exercise. In one study, Sekir and Gür ${ }^{14}$ used a simple 6-week, twice per week multi-station proprioceptive exercise programme to improve postural control, functional capacity and knee pain among 22 persons with bilateral knee OA. In another 8-week, 3 times per week pilot study ${ }^{15}$ it was found that KBA alone improved the pain, stiffness and physical function of subjects with knee OA equally as well as a strength training programme.

Few studies have compared clinic-based with home-based delivery of rehabilitation exercise for knee $O A,{ }^{16-18}$ and no studies are known to have compared clinic-based versus home-based KBA programmes. It is not yet clear if there is a meaningful difference in outcomes between these two delivery methods. Deyle et al. ${ }^{18}$ noted almost double the improvement in self-reported symptom improvement for clinic (52\%) versus home-based (26\%) subjects in a 4-week exercise programme. However, both groups exceeded a clinically relevant threshold for improvement. ${ }^{19}$ In contrast, other 


\section{TABLE I. Agility and balance exercises}

\begin{tabular}{|c|c|}
\hline Exercise & Description \\
\hline Wedding march & Step forward and slightly to one side with right foot, bring left foot together with right foot, alternate leading foot \\
\hline Backward wedding march & As above, stepping backward \\
\hline High knees march & Walk forward while flexing hip to 90 degrees \\
\hline Side-stepping & $\begin{array}{l}\text { Stand with feet together, step to side with right foot, bring left foot to right; repeat for prescribed number of } \\
\text { steps; lead with left foot and then repeat in opposite direction }\end{array}$ \\
\hline Semi-tandem walk & Walk heel-to-toe with heel of leading foot landing just in front of and medial to great toe of opposite foot \\
\hline Tandem walk & Advanced version of above; leading heel lands directly in front of opposite foot \\
\hline Cross-over walk & Walk forward with each foot landing across midline of body \\
\hline Modified grapevine & $\begin{array}{l}\text { Step to side with right foot, bring left foot behind right, step to side with right, bring left in front of right; repeat for } \\
\text { prescribed number of steps; change leading foot and repeat in opposite direction }\end{array}$ \\
\hline Toe walking & Walk forward on toes \\
\hline Heel walking & Walk forward on heels \\
\hline Static balance & Stand on one foot for prescribed period of time \\
\hline Dynamic balance & As above, with the addition of small, rapid bouncing movements \\
\hline
\end{tabular}

investigators have found no differences in efficacy when comparing clinic with home-based exercise interventions for knee OA. ${ }^{16,17}$ The current investigators are engaged in a larger home-based study of KBA efficacy among persons with knee OA. A pilot study ${ }^{15}$ had demonstrated the efficacy of KBA in a clinic setting, but it was not known if similar effects would be seen in a home-based programme. Thus, the purpose of this preliminary study was to compare the efficacy of a knee OA-specific KBA exercise programme delivered in a clinic-based versus a home-based setting.

\section{Methods}

\section{Participants}

All participants $(N=12)$ had physician-diagnosed symptomatic knee $\mathrm{OA}$, reported knee pain on most days of the prior month, met a minimum score for physical function difficulties and were free of other rheumatic disease. Participants were excluded if they had been engaged in a leg exercise programme in the previous 6 months, had an injection in either knee in the previous 30 days, a hip or knee joint replacement, or an unresolved balance disorder. All participants obtained written clearance for exercise from their physicians. For the present investigation, six participants were drawn from each of two larger studies, one using clinic-based (CB) KBA exercise, the other home-based (HB) KBA. Both groups consisted of four women and two men, and all participants had been randomly assigned to the KBA condition. Mean age of $\mathrm{CB}(N=6)$ and $\mathrm{HB}(N=6)$ was $63.3 \pm 12.5$ and $76.5 \pm 11.6$ years, respectively. Body mass index (BMI) of $\mathrm{CB}$ and $\mathrm{HB}$ was $35.7 \pm 11.69$ and $25.2 \pm 2.21 \mathrm{~kg} / \mathrm{m}^{2}$, respectively. The CB study was approved by the BayCare Pasco-Pinellas Institutional Review Board (Clearwater, Florida, USA), and the HB study was approved by the Barry University Institutional Review Board (Miami Shores, Florida, USA). Ethical standards of each board were followed and all participants signed a written informed consent form.

\section{Testing protocols}

The physical function (PF) sub-scale of the Western Ontario and McMaster University (WOMAC) osteoarthritis scale ${ }^{20}$ was the primary outcome measure. WOMAC consists of three symptom sub-scales of pain ( 0 - 20 points), stiffness ( 0 - 8 points), and PF ( 0 - 68 points $)$ and a total score which is a summation of the sub-scales. Ancillary tests included the human activity profile (HAP), ${ }^{21}$ get up \& go (GUG), stair climb and stair descent. HAP is a self-report survey that measures community based maximal and average physical activity. GUG required a participant to rise from a chair and walk a distance of 15 meters as fast as possible. The best time of three GUG trials was recorded. The two stair tests timed a participant first ascending (one trial) and then descending (one trial) a staircase of 10 steps. To assess knee stability, participants responded to a question from the knee outcome survey - activities of daily living scale (KOS-ADLS) ${ }^{22}$ addressing this factor. Paired $t$-tests were conducted to test for differences $(p<0.05)$ from baseline to 8-week follow-up within each of the two conditions. Unpaired t-tests were used to test for differences in outcomes $(p<0.05)$ between conditions.

\section{Exercise interventions}

Each $C B$ session was led by one of two exercise physiologists, trained in the study protocols by the lead investigator. The CB procedures have been previously described. ${ }^{15}$ The lead investigator, an exercise physiologist, worked one-to-one for the first three HB sessions and provided participants with written/pictorial instructions for unsupervised sessions. The investigator followed up by telephone or e-mail, and again in person at weeks 4 and 6 for refresher sessions. Both $\mathrm{CB}$ and $\mathrm{HB}$ consisted of three 30 -minute sessions per week for 8 weeks ( 24 sessions). KBA exercises are described in Table I. The exercise programmes were individualised for each participant's tolerance and abilities within the framework of the overall programme. That is, fewer steps or balance time and/or repetitions would be assigned on a given exercise for a participant who reported increased pain or demonstrated difficulty with that exercise compared with other participants, and in some cases the particular activity would be modified or eliminated. In this way, the programmes were kept both safe and challenging for each participant. In no case was it necessary to modify or eliminate more than one exercise for a given par- 
TABLE II. Ancillary outcomes, change scores versus baseline

\begin{tabular}{|c|c|c|c|c|c|c|c|c|}
\hline Variable $^{\dagger}$ & $\begin{array}{l}\text { Clinic-based } \\
\text { Mean (SD) }\end{array}$ & $\begin{array}{c}\% \\
\text { Change* }\end{array}$ & $p$ & $\mathbf{N}$ & $\begin{array}{l}\text { Home-based } \\
\text { Mean (SD) }\end{array}$ & $\begin{array}{c}\% \\
\text { Change* }^{*}\end{array}$ & $p$ & $N$ \\
\hline 15-m GUG (s) & $-1.51(1.45)$ & 14 & 0.039 & 51 & $-0.58(1.41)$ & 5 & 0.233 & 4 \\
\hline 10-Stair climb (s) & $-1.70(3.14)$ & 22 & 0.146 & 51 & $-1.13(2.73)$ & 17 & 0.272 & 3 \\
\hline 10-Stair descent (s) & $-3.79(4.89)$ & 38 & 0.079 & 51 & $-1.03(2.25)$ & 13 & 0.255 & 3 \\
\hline HAP MAS & $3.16(2.78)$ & 4.5 & 0.019 & 61 & $3.80(12.1)$ & 17 & 0.261 & 5 \\
\hline HAP AAS & $3.16(4.87)$ & 5 & 0.086 & 61 & $7.50(13.2)$ & 13 & 0.169 & 4 \\
\hline \multicolumn{9}{|l|}{ * Rounded } \\
\hline \multicolumn{9}{|c|}{$\begin{array}{l}\text { No between groups differences were found }(p>0.05) \\
\text { GUG = get up \& go walk; HAP MAS = Maximum Activity Score: 'highest oxygen-demanding activity that the respondent still performs'; AAS = Adjusted Activity Score: 'a measure of } \\
\text { usual daily activities' (15). }\end{array}$} \\
\hline
\end{tabular}

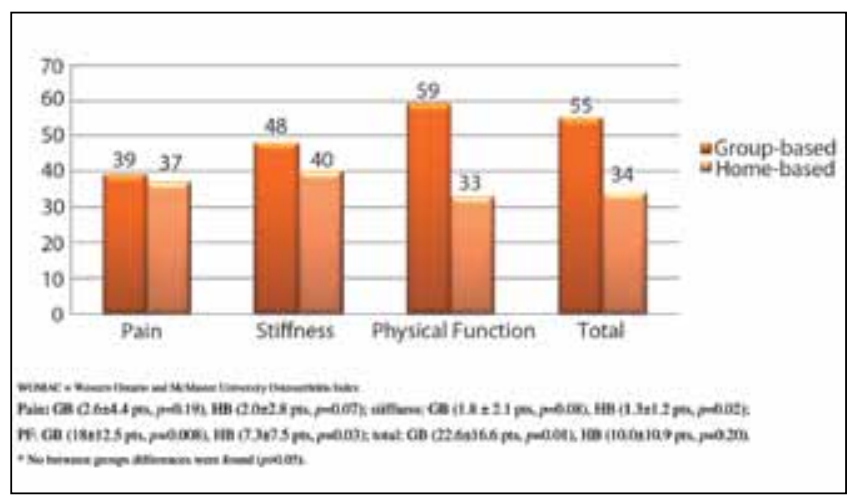

Fig. 1. WOMAC improvements at 8 weeks expressed in \% change.

ticipant. Compliance with the exercise prescription was determined by exercise logs for both the $\mathrm{CB}$ and HB programmes. Participants recorded the number of steps completed for each agility exercise and the time and repetitions of the balance exercises.

\section{Results}

Adherence was $94 \%$ for both conditions. WOMAC results are summarised in Fig. 1. WOMAC PF improved in both $\mathrm{CB}(18 \pm 12.5$ points, $p=0.008)$ and HB $(7.3 \pm 7.5$ points, $p=0.03)$ at the 8 -week follow-up. Ancillary results are presented in Table II. For both WOMAC and ancillary outcomes, CB improvements were generally greater, but these differences did not reach statistical significance.

\section{Discussion}

Given the high lifetime risk and increasing incidence of symptomatic knee $\mathrm{OA}$, and the important role of exercise in mitigating symptoms, it is important to find low-cost, easy-to-administer exercise interventions. While patient-directed home-based programmes should be less expensive than clinician-directed supervised programmes, there has been surprisingly little research comparing the efficacy of the two approaches. We took the opportunity to compare our participants who had completed a KBA training programme in clinic-based exercise physiologist-led sessions with those who completed the same programme as individuals at home with only three supervised familiarisation and two supervised follow-up sessions. While there is some evidence for the efficacy of KBA training to reduce knee OA symptoms in a clinical setting, it was unknown if similar effects would be seen in a home-based semi-supervised setting.
Given the low power of this study (small sample and large standard deviations), our positive within groups WOMAC results indicate a large effect size and are encouraging in terms of efficacy. Large standard deviations are not unexpected in a small group with a broad range of ages (45 - 80 years) and varying functional limitations (though all had the mobility to safely participate in the exercise programmes). Note that conclusions cannot be stated for some ancillary functional tests due to the small numbers in some cells. This was due to some subjects, mostly in the home-based study, being unavailable for follow-up testing. These subjects did return the paper-and-pencil surveys, however.

Our results compliment those of Chamberlain et al. ${ }^{16}$ and Reeder et al. ${ }^{17}$ These investigators found virtually no difference in functional improvement for older persons with knee $O A^{16}$ or chronic health conditions including $\mathrm{OA}^{17}$ when comparing exercise programmes delivered in clinic-based or home-based settings. Other investigators $\left(\right.$ Deyle ${ }^{18}$ ) found a clinic-based knee OA exercise programme (supplemented with home-based exercise and manual therapy) superior to a home-based exercise programme. However, at a one-year follow-up there was no difference between the clinic- and home-based subjects' outcomes, presumably because all subjects continued home-based exercises per the authors. Their clinic-based intervention was noted to be substantially more expensive than the home-based intervention. Note that none of the above investigators employed a KBA programme.

Consistent with a case study ${ }^{11}$ and three published clinical trials, ${ }^{13-15}$ our results indicate that 8 weeks of 3 times per week KBA training appears effective for treating knee OA symptoms. In our comparison, KBA appears to be effective whether delivered in a clinic-based or home-based programme. In addition, both delivery methods resulted in a high adherence rate $(94 \%)$. Similar to Deyle et al., ${ }^{18}$ we did note a higher percentage improvement in total WOMAC score for CB $(55 \%)$ versus HB (34\%). However, we found no statistical differences in change scores between groups, and both groups' WOMAC changes exceeded an established minimal clinically important difference of $20 \%{ }^{19}$ Given the potential cost savings and ease of delivery of home-based exercise interventions for knee OA symptoms, this is a subject worthy of further investigation.

\section{Conclusion}

Our results indicate that KBA exercise taught by an exercise physio logist is effective for improving the symptoms of persons with knee 
OA whether delivered in a supervised clinic-based or a semi-supervised home-based programme. Future research studies with greater statistical power are needed to confirm or refute our efficacy and equivalency findings.

\section{Support}

This research was supported by a product grant from The TheraBand $®$ Academy, which provided the stability trainers.

\section{REFERENCES}

1. Murphy L, Schwartz TA, Helmick CG, et al. Lifetime risk of symptomatic knee osteoarthritis. Arthritis Rheum 2008;59:1207-1213 (doi: 10.1002/ art.24021).

2. Dillon CF, Rasch EK, Gu Q, Hirsh R. Prevalence of knee osteoarthritis in the United States: Arthritis data from the third National Health and Nutrition Examination Survey 1991-94. J Rheumatol 2006;33:2271-2279.

3. Australian Institute of Health and Welfare. A snapshot of arthritis in Australia 2010. Arthritis Series No.13, Cat. No. PHE126. Canberra: AlHW; 2010.

4. Kurtz S, Ong K, Lau E, Mowat F, Halpern M. Projections of primary and revision hip and knee arthroplasty in the United States from 2005 to 2030. J Bone Joint Surg Am 2007;89(4):780-785.

5. Robertsson O, Dunbar MJ, Knutson K, Lidgren L. Past incidence and future demand for knee arthroplasty in Sweden: a report from the Swedish Knee Arthroplasty Register regarding the effect of past and future population changes on the number of arthroplasties performed. Acta Orthop Scand 2000;71(4):376-80.

6. Zhang W, Nuki G, Moskowitz RW, et al OARSI recommendations for the management of hip and knee osteoarthritis: part III: Changes in evidence following systematic cumulative update of research published through January 2009. Osteoarthritis Cartilage 2010;18:476-499.

7. Taylor JB. Lower extremity perturbation training. Strength Cond J 2011;33 (2):76-83.

8. Hubley-Kozey C, Deluzio K, Dunbar M. Muscle co-activation patterns during walking in those with severe knee osteoarthritis. Clin Biomech 2008;23;71-80.

9. Lewek MD, Rudolph KS, Snyder-Mackler L. Control of frontal plane knee laxity during gait in patients with medial compartment knee os- teoarthritis. Osteoarthritis and Cartilage 2004;12:745-751 (doi:10.1016/j. joca.2004.05.005)

10. Rudolph KS, Schmitt LC, Lewek MD. Age-related changes in strength, join laxity, and walking patterns: are they related to knee osteoarthritis? Phys Ther 2007;87(11):1422-1432

11. Fitzgerald GK, Childs JD, Ridge TM, Irrgang JJ. Agility and perturbation training for a physically active individual with knee osteoarthritis. Phys Ther 2002;82:372-382.

12. Bennell K, Hinman R. Exercise as a treatment for osteoarthritis. Curr Opin Rheumatol 2005:17:643-640.

13. Diracoglu D, Aydin R, Baskent A, Celik A. Effects of kinesthesia and balance exercises in knee osteoarthritis. J Clin Rheumatol 2005;11:303-310.

14. Sekir U, Gur H. A multi-station proprioceptive exercise program in patients with bilateral knee osteoarthritis: functional capacity, pain and sensoriomotor function. A randomized controlled trial. J Sports Sci Med 2005;4:590603.

15. Rogers MW, Tamulevicius N, Coetsee MF, Curry BF, Semple SJ. Knee osteoarthritis and the efficacy of kinesthesia, balance \& agility exercise training: a pilot study. Int J Exerc Sci 2011;4(2),Article 5.

16. Chamberlain MA, Care G, Harfield B. Physiotherapy in osteoarthrosis of the knees. A controlled trial of hospital versus home exercises. Int Rehabil Med: 1982;4:101-106.

17. Reeder BA, Chad KE, Harrison EL, et al. Saskatoon in motion: class- versus home-based exercise intervention for older adults with chronic health conditions. J Phys Act Health 2008:5:74-87.

18. Deyle GD, Allison SC, Matekel RL, et al. Physical therapy treatment effectiveness for osteoarthritis of the knee: a randomized comparison of supervised clinical exercise and manual therapy procedures versus a home exercise program. Phys Ther 2005;85:1301-1317.

19. Barr S, Bellamy N, Buchanan WW, et al. A comparative study of signal versus aggregate methods of outcome measurement based on the WOMAC Osteoarthritis Index. J Rheumatol 1994;21:2106-2112.

20. Bellamy N, Buchanan WW, Goldsmith $\mathrm{CH}$, Campbell J, Stitt LW. Validation study of WOMAC: a health status instrument for measuring clinically important patient relevant outcomes to antirheumatic drug therapy in patients with osteoarthritis of the hip or knee. J Rheumatol 1988;15:1833-1840.

21. Fix AJ, Daughton DM. Human activity profile professional manual. Lutz, FI: Psychological Assessment Resources, Inc; 1988.

22. Irrgang JJ, Snyder-Mackler L, Wainner RS, Fu FH, Harner CD. Development of a patient-reported measure of function of the knee. J Bone Joint Surg Am 1998;80(8):1132-1145 